\title{
As the Story Unfolds
}

\author{
Garth W Strohbehn, MD, MPhil1,2*, Sanjay Saint, MD, MPH'3,45, David L Grinblatt, MD², \\ Justine Moe, MD, DDS ${ }^{6}$, Gurpreet Dhaliwal, MD7,8
}

This icon represents the patient's case. Each paragraph that follows represents the discussant's thoughts.

${ }^{1}$ Section of Hematology/Oncology, University of Chicago, Chicago, Illinois; ${ }^{2}$ Kellogg Cancer Center, NorthShore University HealthSystem, Evanston, Illinois; ${ }^{3}$ Medicine Service, VA Ann Arbor Healthcare System, Ann Arbor, Michigan; ${ }^{4}$ Center of Innovation, VA Ann Arbor Healthcare System, Ann Arbor, Michigan; ${ }^{5}$ Department of Internal Medicine, University of Michigan Medical School, Ann Arbor, Michigan; ${ }^{6}$ Section of Oral and Maxillofacial Surgery, Department of Surgery, University of Michigan Medical School, Ann Arbor, Michigan; ${ }^{7}$ Medical Service, San Francisco Veterans Affairs (VA) Medical Center, San Francisco, California; ${ }^{8}$ Department of Medicine, University of California, San Francisco, San Francisco, California.

$\square^{\infty}$

A 78-year-old woman presented to the ambulatory care clinic for a painful tongue mass. She noticed the mass 2 months prior to presentation, and it had not grown in the interim. She had left-sided jaw pain when opening her mouth and persistent left-sided otalgia.

In the evaluation of tongue masses, ulcerations, or other surface abnormalities, exclusion of squamous cell carcinoma is the top priority. Additional tongue surface abnormalities include benign lesions such as geographic tongue, inflammatory conditions such as lichen planus, and infections such as syphilis.

The ear and jaw pain may reflect metastatic spread, neural invasion, or referred pain from the tongue. A vasculitis with predilection for the head, such as giant cell arteritis, could present with oral and ear pain. Jaw pain with mastication could reflect jaw claudication, but pain upon mouth opening is more commonly explained by temporomandibular joint dysfunction.

The patient had hypertension, hyperlipidemia, chronic kidney disease (estimated glomerular filtration rate of $42 \mathrm{~mL} / \mathrm{min}$ ), and diabetes mellitus. Four months prior she was diagnosed with a chronic obstructing left renal calculus on ultrasonography to evaluate chronic kidney disease. Two months prior heart failure with preserved ejection fraction was diagnosed. Stress cardiac magnetic resonance imaging (MRI) demonstrated normal ejection fraction, asymmetric septal hypertrophy, and stress-induced subendocardial perfusion defect. Her medications were metoprolol, lisinopril, simvastatin, meloxicam, and aspirin. She never used tobacco and did not consume alcohol. She was born in the Philippines and emigrated to the United States 15 years ago. She had not experienced fever, chills, hearing loss, tinnitus, cough, dysphonia, neck swelling, or joint pain. She had lost $3 \mathrm{~kg}$ in the previous 4 months.

*Corresponding Author: Garth W Strohbehn, MD, MPhil; Email: gstrohbe@ umich.edu; Twitter: @garthstrohbehn.

Published online first June 11, 2021

Received: April 22, 2020; Revised: July 31, 2020; Accepted: August 6, 2020

๑) 2021 Society of Hospital Medicine DOI 10.12788/jhm.3518
The absence of tobacco and alcohol use reduces the probability of a squamous cell carcinoma, although human papillomavirus-associated squamous cell carcinoma of the tongue remains possible. Asymmetric septal hypertrophy is characteristic of hypertrophic cardiomyopathy or an infiltrative cardiomyopathy. Sarcoidosis can affect the heart and can account for the renal calculus (via hypercalcemia). Amyloid light-chain (AL) amyloidosis could involve the heart and the tongue, although in amyloidosis the cardiac MRI typically displays late gadolinium enhancement and ventricular wall thickening. The absence of tinnitus or hearing loss suggests that the left-sided otalgia is referred pain from the tongue and oral cavity rather than a primary otologic disease (eg, infection).

On physical examination, temperature was $37.2{ }^{\circ} \mathrm{C}$, heart rate was 88 beats per minute, blood pressure was $134 / 62 \mathrm{~mm} \mathrm{Hg}$, and oxygen saturation was $99 \%$ while breathing ambient air. The patient's weight was $40.4 \mathrm{~kg}$ (body mass index of $19.26 \mathrm{~kg} / \mathrm{m}^{2}$ ). Intraoral examination revealed induration of the bilateral tongue, an erosive $1-\mathrm{cm}$ pedunculated mass of the left dorsum, rough white coating on the right dorsum, and fullness of the right lateral surface with an erosion abutting tooth \#2. The right submandibular salivary gland was firm. The otoscopic examination and remainder of the head and neck examination were normal. There was no cervical, supraclavicular, or axillary adenopathy. The cranial nerve, cardiovascular, pulmonary, abdominal, and skin examinations were normal.

The left-sided lingual mass and right-sided lingual erosion likely arise from the same process. Both are compatible with an infection (eg, syphilis or tuberculosis), cancer, autoimmune disease (eg, Crohn's disease or sarcoidosis), or an infiltrative disease such as amyloidosis. Leukoplakia could reflect a candidal infection, dysplasia, squamous cell carcinoma, oral hairy leukoplakia, or hyperkeratosis. The isolated submandibular salivary gland could reflect sialadenitis from chronic salivary duct obstruction or a primary neoplasm, but more likely is caused by the same process causing the tongue abnormalities. 

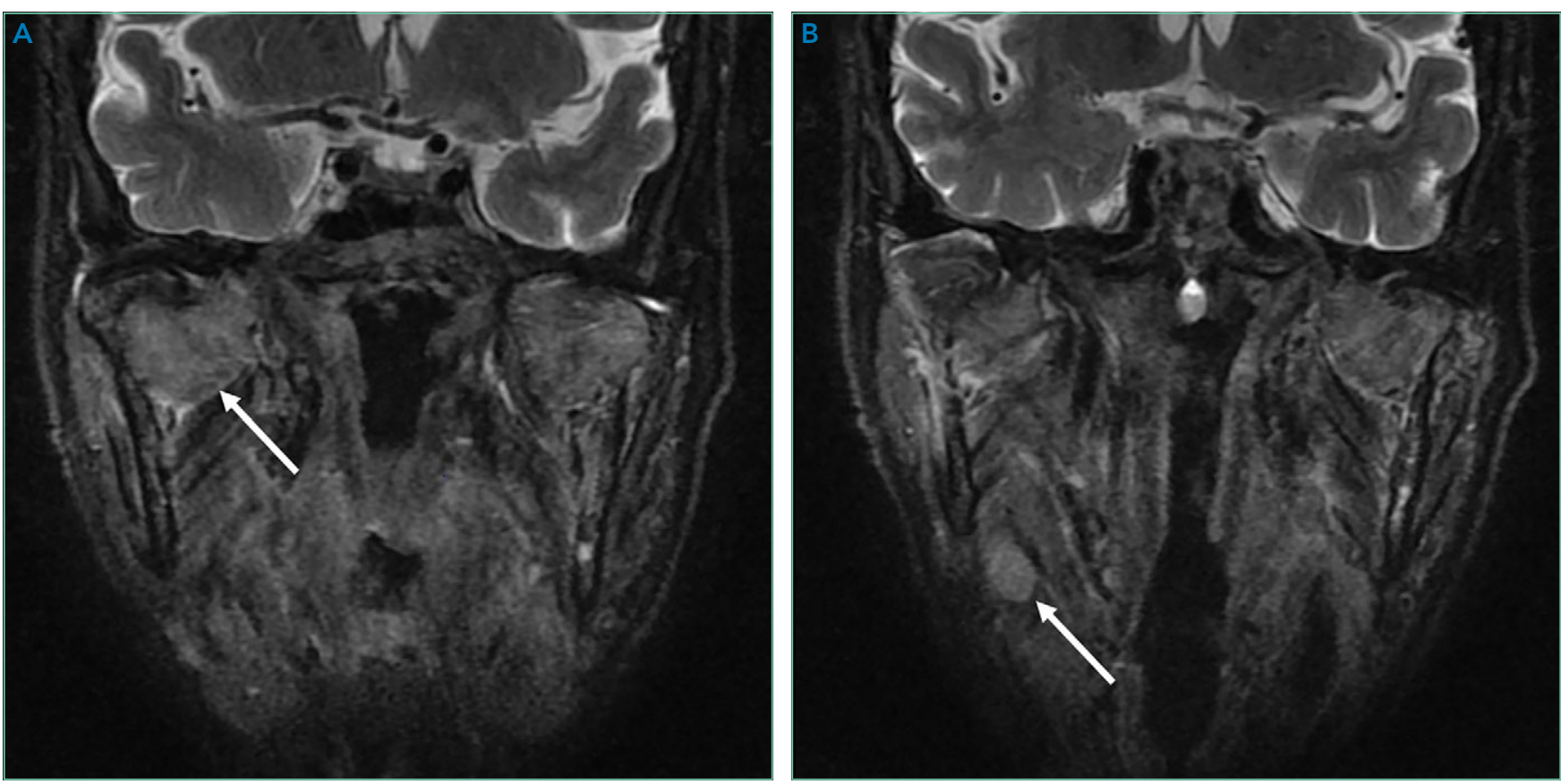

FIG 1. Magnetic Resonance Imaging of Head, Face, and Neck. (A) T2-weighted magnetic resonance imaging demonstrating hyperintensity and prominence of right lateral pterygoid muscle (arrow) and (B) contrast enhancement of submandibular gland (arrow). Oral tongue, base of tongue, and vestibulocochlear system were negative for masses.

The white blood cell count was $8,600 / \mu \mathrm{L}$; hemoglobin, $11.5 \mathrm{~g} / \mathrm{dL}$; mean corpuscular volume, $102.5 \mathrm{fL}$; and platelet count, $270,000 / \mu \mathrm{L}^{3}$. Serum sodium was $141 \mathrm{mEq} / \mathrm{L}$; potassium, $4.1 \mathrm{mEq} / \mathrm{L}$; chloride, $101 \mathrm{mEq} / \mathrm{L}$; bicarbonate, 30 $\mathrm{mEq} / \mathrm{L}$; blood urea nitrogen, $19 \mathrm{mg} / \mathrm{dL}$; creatinine, $0.7 \mathrm{mg} /$ $\mathrm{dL}$; and calcium, $10.4 \mathrm{mg} / \mathrm{dL}$ (reference range, 8.5-10.3). Total serum protein was $7.3 \mathrm{~g} / \mathrm{dL}$ (reference range, 6.0-8.3); albumin was $3.7 \mathrm{~g} / \mathrm{dL}$. Liver biochemistry test results were normal. Serum folate and vitamin $B_{12}$ levels were normal. Serum ferritin was $423 \mathrm{ng} / \mathrm{mL}$ (reference range, 11-306); transferrin saturation, $21.4 \%$ (reference range, $15.0 \%$ $50.0 \%$ ); and total iron-binding capacity, $323 \mu \mathrm{g} / \mathrm{dL}$ (reference range, 261-478). Parathyroid hormone (PTH) was 14 $\mathrm{pg} / \mathrm{mL}$ (reference range, 15-65). HIV antibody was negative.

The calcium level is at the upper range of normal, whereas the PTH level is at the lower range of normal. The differential diagnosis for PTH-independent hypercalcemia includes hypercalcemia of malignancy and granulomatous disease such as sarcoidosis. Mild hypercalcemia could contribute to the nephrolithiasis. The iron studies exclude iron deficiency and are not suggestive of anemia of chronic disease. The triad of mild hypercalcemia, cardiomyopathy, and anemia is compatible with AL amyloidosis (perhaps with associated multiple myeloma) or sarcoidosis; both disorders can present as a mass. Imaging of the head and neck and biopsy of the tongue mass are the next steps.

\section{The left dorsal tongue mass was excised in clinic. Histo-} pathology revealed ulcerated squamous mucosa with inflammatory changes but no malignancy. Imaging of the head and neck was scheduled.

Neither cancer or granulomas were detected, but inadequate sampling or staining must be considered. Inflammatory changes are compatible with infection, autoimmunity, and cancer; the latter can feature reactive changes that obscure the malignant cells. The absence of granulomas lowers, but does not eliminate, the possibility of sarcoidosis, tuberculosis, fungal infection, and granulomatosis with polyangiitis. Actinomycosis is an invasive orofacial infection that disregards anatomic boundaries and is characterized by inflammatory histology; although infection of the tongue is possible, infection of the jaw and face is more typical. Immunoglobulin G4-related disease can present as an inflammatory and invasive disorder; however, the characteristic histopathologic findings (lymphoplasmacytic infiltrate, fibrosis, and phlebitis) are absent.

Culture of the tissue for mycobacteria or fungi (she is at increased risk for both given her previous residency in the Philippines) could increase the diagnostic yield. Another biopsy of the tongue or an adjacent structure-guided by imagingmay provide a more diagnostic tissue sample.

MRI of the head and neck demonstrated hyperintense signal and prominence of the right lateral pterygoid muscle (Figure $1 \mathrm{~A}$ ) and slight enlargement of a right submandibular gland (Figure 1B). No tongue abnormalities were identified. Radiograph of the chest did not reveal infiltrates, masses, or lymphadenopathy. 
The absence of the tongue mass on the MRI likely reflects excision of the mass at the time of biopsy. The signal enhancement in the right lateral pterygoid muscle and submandibular gland is suggestive of an infiltrative process. Infiltration of the right lateral pterygoid muscle may also explain the patient's pain when opening her mouth. Infiltrative processes can be neoplastic (eg, salivary gland tumor, sarcoma, lymphoma), infectious (eg, mycobacterial or fungal), cellular (eg, histiocytes, mast cells, plasma cells, eosinophils, granulomas), or related to inert substances such as amyloid or iron.

Seven weeks later, the patient presented to the hospital for scheduled percutaneous nephrolithotomy of the obstructing renal calculus. The physical examination was unchanged. The complete blood count and metabolic panel were unchanged apart from hemoglobin of $9.9 \mathrm{~g} / \mathrm{dL}$ and calcium of $11.5 \mathrm{mg} / \mathrm{dL}$. Coagulation studies were within normal limits.

A percutaneous nephroureteral stent was placed under conscious sedation. The patient then underwent rapid sequence induction of general anesthesia for the nephrolithotomy with fentanyl, propofol, and rocuronium. Within minutes of initiating mechanical ventilation, severe periorbital and perioral edema, copious oral cavity bleeding, and bilateral periorbital purpura occurred. Sugammadex (neuromuscular blockade reversal) and dexamethasone were administered. Examination of the oral cavity was limited by the brisk bleeding; the right sided tongue erosion was unchanged.

Bleeding is caused by thrombocytopenia, thrombocytopathy, coagulopathy, or disruption of vessel integrity. Oral cavity bleeding could arise from the tongue ulceration, but could also reflect pulmonary, nasal, or gastrointestinal hemorrhage. Angioedema arises from mast cell- or bradykinin-mediated pathways; mast cell degranulation may have been precipitated by the anesthetic agents, opiate, or a material in the nephroureteral stent.

The edema and bleeding are temporally related to multiple medications and mechanical ventilation. A latent bleeding diathesis may have manifested in the setting of increased tissue hydrostatic pressure or vessel permeability. Amyloidosis can lead to vessel fragility and coagulopathy, and periorbital bleeding is characteristic of $\mathrm{AL}$ amyloidosis.

The hypercalcemia, now more pronounced, raises concern for malignancy (including multiple myeloma) and granulomatous diseases like sarcoidosis, mycobacterial infections, and fungal infections. The declining hemoglobin could be explained by chronic blood loss, hemolysis, anemia of chronic disease, or a bone marrow process.

The cardiomyopathy, bleeding disorder, and multifocal disease in the oral cavity can be explained by $\mathrm{AL}$ amyloidosis; the hypercalcemia suggests concomitant multiple myeloma.

At the time of the bleeding event, the partial thromboplastin time, prothrombin time, and fibrinogen were within the reference ranges. Factor $X$ activity level was normal. No schistocytes were observed on peripheral blood smear. Immu-

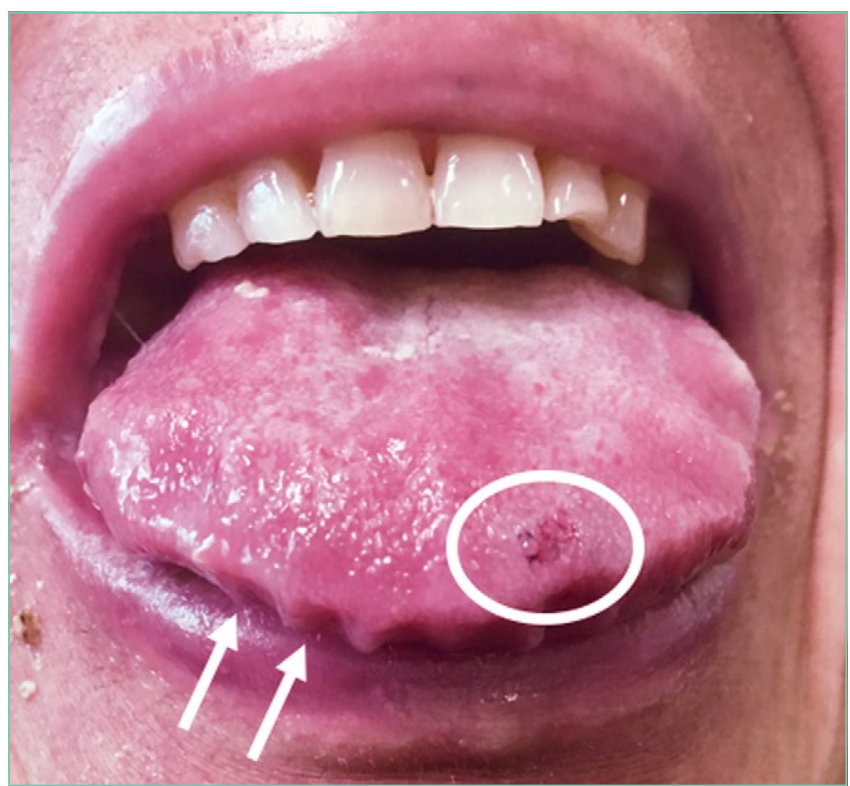

FIG 2. Image of Patient's Tongue at Time of Hematology Consultation. The patient's tongue demonstrated an anterior, dorsal lesion (circle). The tongue is also crenated ("scalloped") (arrows)

noglobulin $\mathrm{G}$ level was $1,425 \mathrm{mg} / \mathrm{dL}$ (reference range, 639$1,349) ; \lg A$ and $\lg M$ levels were within the reference range. Serum lambda free light chains were $151.78 \mathrm{mg} / \mathrm{dL}$ (reference range, $0.46-2.71)$, and the ratio of kappa to lambda light chains was 0.01 (reference range, 0.49-2.54). Serum protein electrophoresis and immunofixation demonstrated a monoclonal paraprotein (lgG lambda) level of $1.2 \mathrm{~g} / \mathrm{dL}$. Congo red staining of the previously excised left dorsal tongue mass was negative for apple-green birefringence. Reexamination of the oral cavity revealed macroglossia and scalloping of the tongue (Figure 2).

Scalloping is characteristic of an infiltrative disorder that enlarges the tongue (macroglossia) and deforms its edges, which encounter the teeth. Macroglossia is seen in $\mathrm{AL}$ amyloidosis, acromegaly, and hypothyroidism. A monoclonal light chain, especially a lambda light chain, is characteristic of $A L$ amyloidosis. The Congo red stain results can support the diagnosis when positive, but it has limited sensitivity. The tongue specimen can be sent for immunohistochemistry or mass spectrometry to evaluate for light chain deposition. A bone marrow biopsy can demonstrate a clonal plasma cell population. $\mathrm{AL}$ amyloidosis with concomitant multiple myeloma is the most likely diagnosis.

Bone marrow aspiration and core biopsy demonstrated $30 \%$ lambda-restricted plasma cells (Figure 3A-C). Congo red staining demonstrated apple-green birefringence of the bone marrow microvasculature (Figure 3D). Skeletal survey demonstrated widespread lytic bone disease involving the calvarium (Figure 4A), left humerus (Figure 4B), and left scapula (Figure 4B). Based on the monoclonal paraprotein, more than $10 \%$ monoclonal plasma cells, skeletal lesions, and hypercalcemia, she was diagnosed with IgG lambda multiple myeloma. Based on apple-green birefringence in 

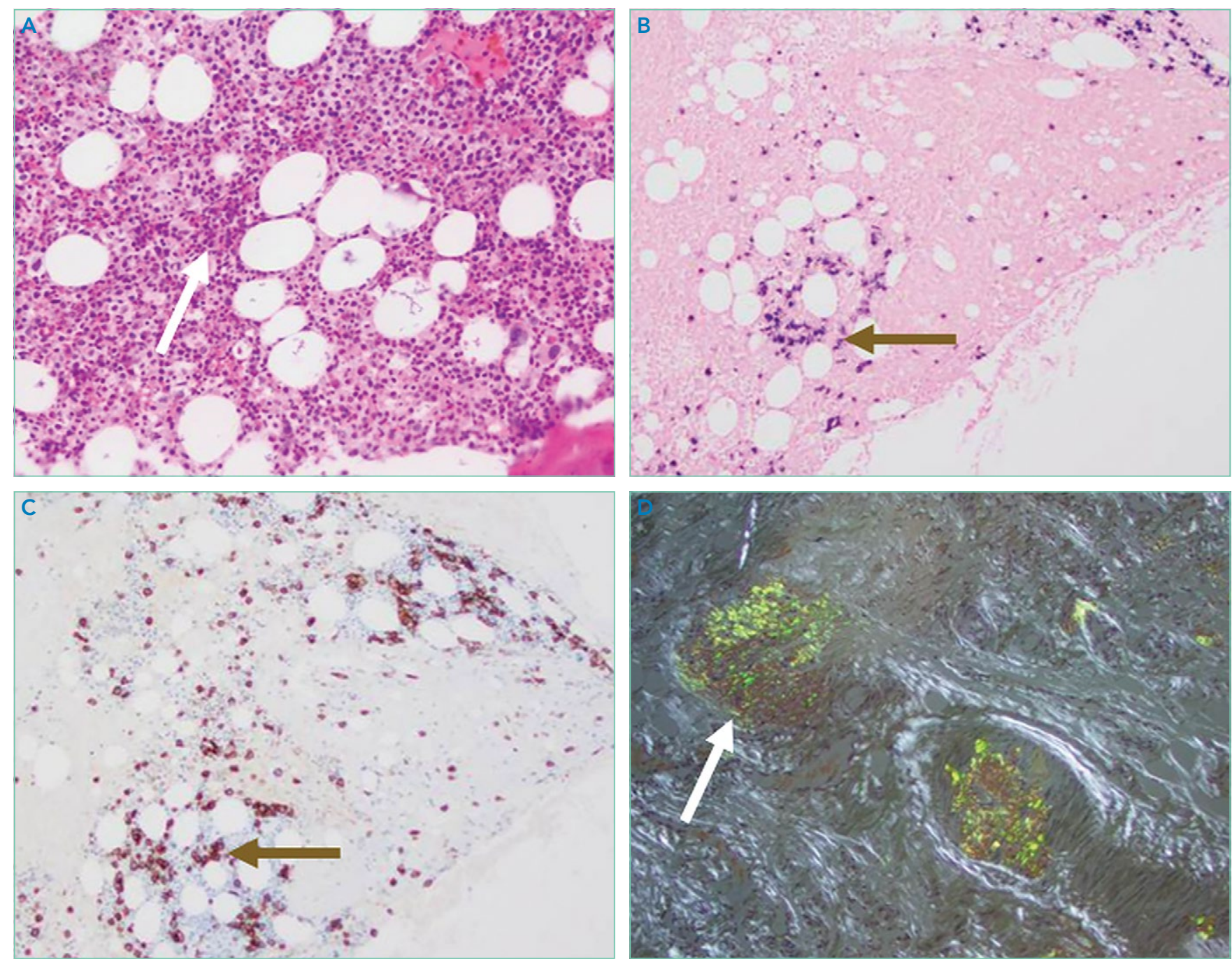

FIG 3. Bone Marrow Biopsy. (A) Bone marrow core biopsy demonstrated large clusters of plasma cells throughout the bone marrow (white arrow). (B) Staining for CD138 showed plasma cell clusters (brown arrow) that were (C) lambda-light chain-restricted (brown stain, brown arrow). (D) Congo red stain demonstrated perivascular apple-green birefringence (white arrow) under polarized light.

the bone marrow and macroglossia, she was diagnosed with $\mathrm{AL}$ amyloidosis. The cardiac MRI findings were compatible with $\mathrm{AL}$ amyloidosis. ${ }^{1}$

After three cycles of bortezomib and dexamethasone therapy to concurrently treat $\mathrm{AL}$ amyloidosis and multiple myeloma, the serum lambda light chain level decreased to $1.49 \mathrm{mg} / \mathrm{dL}$ and the monoclonal paraprotein level decreased to $0.3 \mathrm{~g} / \mathrm{dL}$. The calcium level was $9.8 \mathrm{mg} / \mathrm{dL}$, and the hemoglobin level was $11.7 \mathrm{~g} / \mathrm{dL}$. The patient's tongue pain resolved, allowing for improved oral intake and a 5.7-kg weight gain. The patient underwent nephrolithotomy 4 months after her initial presentation. She resumed an active lifestyle and recently traveled to visit relatives in the Philippines.

\section{DISCUSSION}

Oral diseases affect general health and quality of life and can be a harbinger of systemic disease. Tooth loss, caries, periodontal disease, and poorly fitting dentures commonly affect speech and nutrition. ${ }^{2}$ These common outpatient oral health issues can be the driving force for hospital admissions; for example, caries and periodontal disease can lead to suppurative odontogenic infection, endocarditis, brain abscess, and sepsis.

Tongue ulcerations, masses, and surface abnormalities often require consultation with a dentist or oral and maxillofacial surgeon to exclude squamous cell carcinoma. ${ }^{3}$ Other diagnostic considerations include benign neoplasms, trauma, inflammatory conditions (eg, sarcoidosis), infection (eg, syphilis, tuberculosis), and infiltrative processes such as amyloidosis.

Amyloidosis is a heterogeneous group of diseases caused by deposition of insoluble protein fibrils in tissues. ${ }^{4,5}$ The three most encountered forms of amyloidosis are $A L, A A$, and ATTR. Each form is named after the culprit protein. ${ }^{4} \mathrm{AL}$ amyloidosis arises when a small clonal population of plasma cells in the bone marrow overproduces immunoglobulin light chain monomers. ${ }^{4,6}$ AA amyloidosis develops when the liver produces serum amyloid $A$ protein (an acute phase reactant) in response to a chronic inflammatory condition such as rheumatoid arthritis or chronic intravenous drug injection. ${ }^{4}$ Transthyretin (TTR, also known as "prealbumin") is a te- 

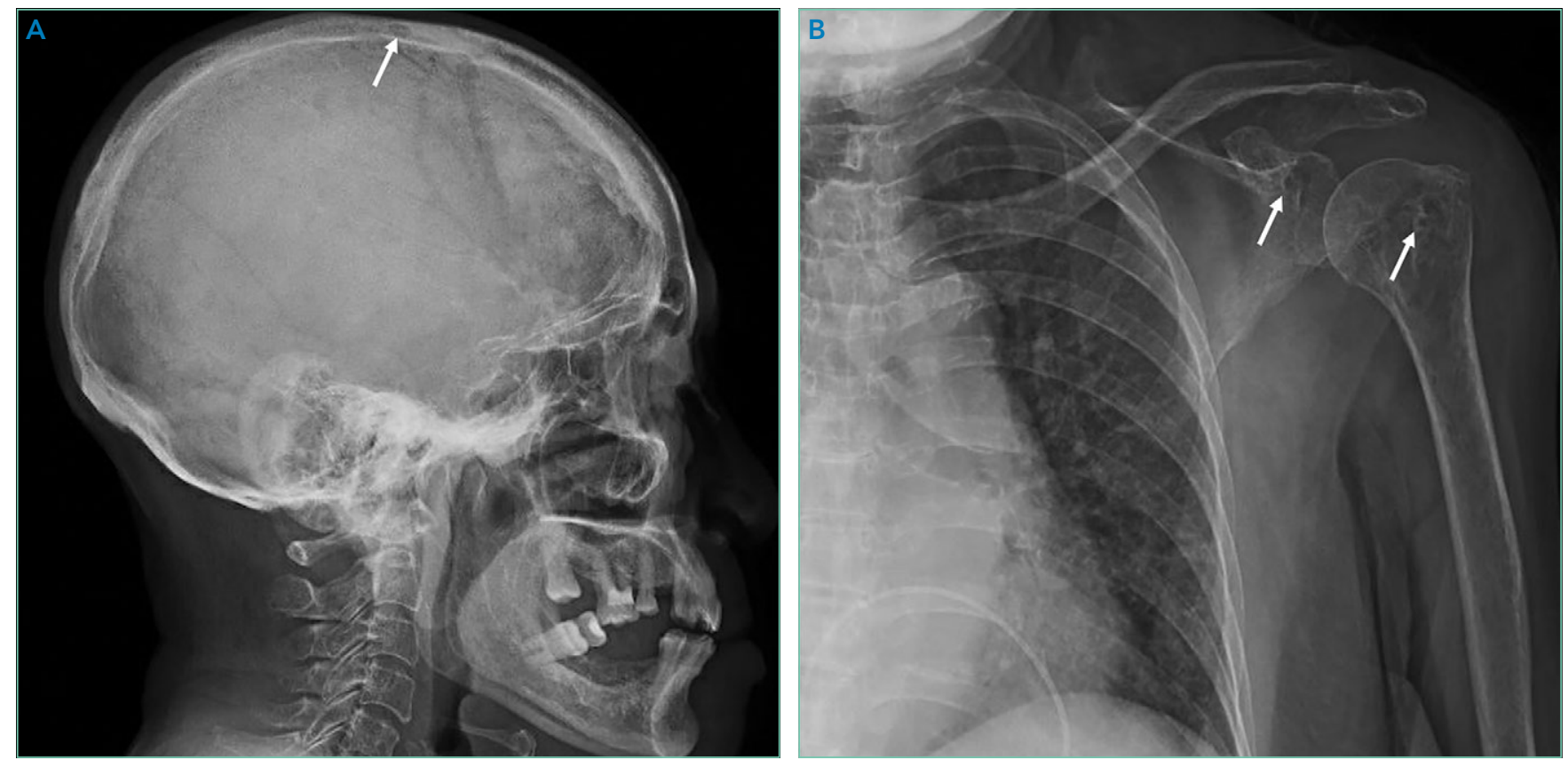

FIG 4. Skeletal Survey. Skeletal survey revealed subtle lytic lesions in the calvarium (A, arrow) and left proximal humerus and scapula (B, arrow on right and left, respectively).

trameric protein that transports thyroxine and retinol; there are two forms of ATTR amyloidosis: hereditary and wild type. Hereditary ATTR amyloidosis develops from agglomeration of misfolded TTR monomers caused by mutations in the TTR gene. Wildtype ATTR amyloidosis is caused by age-related dissociation of the TTR tetramer into its constituent monomers that denature, misfold, and agglomerate into fibrils. ${ }^{5}$ Wild-type ATTR is now recognized as the most common form of amyloidosis, with $25 \%$ of myocardial autopsy specimens of patients 80 years or older demonstrating amyloid. ${ }^{7}$ The estimated incidence of $\mathrm{AL}$ amyloidosis is 10 cases per million person-years. ${ }^{8}$

Each amyloid protein homes in on specific anatomic sites. ${ }^{4}$ Characteristic combinations of organ dysfunction can suggest different forms of amyloidosis. ${ }^{9}$ Cardiac and peripheral nervous involvement (eg, carpal tunnel syndrome) is typical of both hereditary and wild-type ATTR amyloidosis; ATTR amyloidosis does not involve the kidney. ${ }^{4}$ AA amyloidosis most commonly manifests with proteinuria followed by declining glomerular filtration rate; heart failure is rare. ${ }^{4}$ The most common findings in $A L$ amyloidosis are proteinuria, congestive heart failure, and sensory neuropathy. ${ }^{6}$ Gastrointestinal tract and hepatic involvement are each seen in nearly $20 \%$ of patients, and macroglossia is identified in approximately $10 \%$ of those with $A L$ amyloidosis. 6,10

Chronic deposition of amyloid can lead to acute presentations. Approximately $30 \%$ of patients with $\mathrm{AL}$ amyloidosis develop abnormal bleeding. ${ }^{11}$ Amyloid deposition in small blood vessels predisposes them to rupture. Bleeding events can be exacerbated by acquired coagulopathy due to plasma cell dyscrasia-associated thrombocytopenia, amyloid fibril adsorption of factor $X$, or hypofibrinogenemia. ${ }^{11,12}$ Periorbital purpura following minor trauma or transient venous hypertension is characteristic of $A L$ amyloidosis. ${ }^{6,13}$ In this case, positive pressure ventilation and recumbent positioning increased hydrostatic pressure in the head and neck, causing rupture of the infiltrated small vessels around the eyes and in the oral cavity. ${ }^{14}$

Histological demonstration of tissue deposition of amyloid protein is the preferred method for amyloidosis diagnosis. Symptomatic sites or organs with dysfunction or radiologic changes are suitable for biopsy. ${ }^{6}$ If those sites are inaccessible or yield insufficient tissue quantity, abdominal fat pad aspiration or biopsy is indicated. ${ }^{15}$ Apple-green birefringence under polarized light of Congo red-stained tissue is characteristic, with sensitivity and specificity of approximately $80 \%$ and a positive predictive value of $85 \% .{ }^{15}$ Immunoelectron microscopy is often performed simultaneously to confirm the diagnosis and determine the amyloid protein type. ${ }^{4,16}$ Immunoelectron microscopy's sensitivity is approximately $80 \%$, and it has specificity and positive predictive value both approaching $100 \% .{ }^{15}$ Mass spectrometry is particularly useful in cases where the amyloid subtype is not clinically apparent (eg, a patient with an autoimmune condition or chronic infection as well as light chain abnormality). ${ }^{6}$ Cardiac MRI findings that suggest amyloidosis include a thickened left ventricle and late gadolinium enhancement. ${ }^{1}$ ATTR cardiac amyloidosis can be diagnosed using amyloid fibril-binding radiotracer technetium-99m-pyrophosphate scintigraphy; biopsy is often not necessary. ${ }^{1,4}$ Gene sequencing to differentiate between hereditary and wild-type forms of ATTR amyloidosis is beneficial.

The primary objectives of amyloidosis management are to control symptoms and inhibit amyloid protein production. ${ }^{6}$ Outcomes in $\mathrm{AL}$ amyloidosis have improved due to early diagnosis, new chemotherapeutic agents to eradicate the plasma cell clone, and autologous stem cell transplantation. ${ }^{6,17}$ Two new ATTR amyloidosis treatments are RNA interference 
therapies, which prevent TTR messenger RNA translation, and tafamidis, which stabilizes the TTR tetramer and prevents dissociation into its constituent monomers that precipitate in tissues. ${ }^{18}$ Both therapies can improve neuropathy-related quality of life. ${ }^{18}$ Tafamidis slows disease progression and decreases allcause mortality in patients with hereditary and wild-type ATTR cardiac amyloidosis. ${ }^{19}$

Multiple myeloma and $\mathrm{AL}$ amyloidosis are both plasma cell dyscrasias involving the bone marrow, but they represent distinct disease processes with different clinical features. ${ }^{6}$ Multiple myeloma is characterized by marked expansion of a clonal plasma cell population within the bone marrow that aberrantly produces immunoglobulin. Conversely, the clonal plasma cell population responsible for producing the insoluble monoclonal light chain protein in $\mathrm{AL}$ amyloidosis typically constitutes less than $10 \%$ of the bone marrow. ${ }^{20}$ Multiple myeloma and $\mathrm{AL}$ amyloidosis may coexist in the same patient; nearly $15 \%$ of patients with multiple myeloma subsequently develop clinically overt $\mathrm{AL}$ amyloidosis, which portends a poor outcome. ${ }^{20}$

Amyloidosis is a rare group of diseases that arises when misfolded proteins aggregate in vital organs. The typical manifestations-congestive heart failure, neuropathy, chronic kidney disease, bleeding - are nearly always explained by more common conditions. Characteristic manifestations (like macroglossia) or associated diseases (like multiple myeloma) substantially increases the probability of $\mathrm{AL}$ amyloidosis. In a multisystem illness, the most common diseases must be excluded first, but this case reminds us that rare diseases, like amyloidosis, also warrant consideration as the story unfolds.

\section{KEY TEACHING POINTS}

- Different amyloid proteins precipitate in different anatomic sites, which leads to specific multiorgan combinations. The most common amyloidosis, ATTR, tends to manifest as heart failure and peripheral sensory neuropathy, while the constellation of AL amyloidosis includes heart failure, neuropathy, and proteinuria.

- Bleeding occurs in $30 \%$ of patients with $\mathrm{AL}$ amyloidosis. It is precipitated by fragile small blood vessels and exacerbated by acquired coagulopathy from adsorption of coagulation factors.

- Multiple myeloma and AL amyloidosis are both plasma cell dyscrasias involving the bone marrow, but they represent distinct disease processes with different clinical tempos and presentations. Multiple myeloma and $\mathrm{AL}$ amyloidosis may coexist in the same patient; nearly $15 \%$ of patients with multiple myeloma subsequently develop clinically overt $\mathrm{AL}$ amyloidosis.

\section{Acknowledgment}

The authors thank Benjamin A Derman, MD, of the University of Chicago, Chicago, Illinois, for critical review of the manuscript.
Disclosures: Dr Strohbehn reports graduate medical education fellowship support from Abbott Laboratories charitable contributions and pending provisional patent, both outside the submitted work. Dr Saint reports personal fees from Jvion and Doximity and speaking honoraria from ISMIE Mutual Insurance Company, all outside the submitted work. Dr Grinblatt reports speaking honoraria from Alexion Pharmaceuticals and scientific advisory honoraria from Celgene, Astellas, Acceleron, and AbbVie, all outside the submitted work. Dr Moe has nothing to disclose. Dr Dhaliwal reports speaking honoraria from ISMIE Mutual Insurance Company and GE Healthcare, all outside the submitted work.

\section{References}

1. Witteles RM, Bokhari S, Damy T, et al. Screening for transthyretin amyloid cardiomyopathy in everyday practice. JACC Heart Fail. 2019;7(8):709-716. https://doi.org/10.1016/j.jchf.2019.04.010

2. Griffin SO, Jones JA, Brunson D, Griffin PM, Bailey WD. Burden of oral disease among older adults and implications for public health priorities. Am J Public Health. 2012;102(3):411-418. https://doi.org/10.2105/ajph.2011.300362

3. Ernster JA, Sciotto CG, O'Brien MM, et al. Rising incidence of oropharyngeal cancer and the role of oncogenic human papilloma virus. Laryngoscope. 2007;117(12):2115-2128. https://doi.org/10.1097/mlg.0b013e31813e5fbb

4. Wechalekar AD, Gillmore JD, Hawkins PN. Systemic amyloidosis. Lancet. 2016;387(10038):2641-2654. https://doi.org/10.1016/s0140-6736(15)01274-x

5. Riek R, Eisenberg DS. The activities of amyloids from a structural perspective. Nature. 2016;539(7628):227-235. https://doi.org/10.1038/nature20416

6. Gertz MA, Dispenzieri A. Systemic amyloidosis recognition, prognosis, and therapy: a systematic review. JAMA. 2020;324(1):79-89. https://doi. org/10.1001/jama.2020.5493

7. Ruberg FL, Berk JL. Transthyretin (TTR) cardiac amyloidosis. Circulation. 2012;126(10):1286-1300. https://doi.org/10.1161/circulationaha.111.078915

8. Quock TP, Yan T, Chang E, Guthrie S, Broder MS. Epidemiology of AL amyloidosis: a real-world study using US claims data. Blood Adv. 2018;2(10): 1046-1053. https://doi.org/10.1182/bloodadvances.2018016402

9. Papoutsidakis N, Miller EJ, Rodonski A, Jacoby D. Time course of common clinical manifestations in patients with transthyretin cardiac amyloidosis: delay from symptom onset to diagnosis. J Card Fail. 2018;24(2):131-133. https:// doi.org/10.1016/j.cardfail.2017.12.005

10. Shimazaki $\mathrm{C}$, Hata $\mathrm{H}$, lida $\mathrm{S}$, et al. Nationwide survey of 741 patients with systemic amyloid light-chain amyloidosis in Japan. Intern Med. 2018;57(2): 181-187. https://doi.org/10.2169/internalmedicine.9206-17

11. Mumford AD, O'Donnell J, Gillmore JD, Manning RA, Hawkins PN, Laffan M. Bleeding symptoms and coagulation abnormalities in 337 patients with AL-amyloidosis. Br J Haematol. 2000;110(2):454-460. https://doi.org/10.1046/ j.1365-2141.2000.02183.x

12. Choufani EB, Sanchorawala $V$, Ernst $T$, et al. Acquired factor $X$ deficiency in patients with amyloid light-chain amyloidosis: incidence, bleeding manifestations, and response to high-dose chemotherapy. Blood. 2001;97(6): 1885-1887. https://doi.org/10.1182/blood.v97.6.1885

13. Slagel GA, Lupton GP. Postproctoscopic periorbital purpura. Primary systemic amyloidosis. Arch Dermatol. 1986;122(4):464-465, 467-468.

14. Lupton GP. Pneomometry-induced purpura. Arch Dermatol. 1981;117(10):603. https://doi.org/10.1001/archderm.117.10.603a

15. Fernández de Larrea $C$, Verga L, Morbini P, et al. A practical approach to the diagnosis of systemic amyloidoses. Blood. 2015;125(14):2239-2244. https:// doi.org/10.1182/blood-2014-11-609883

16. Vaxman I, Gertz M. Recent Advances in the diagnosis, risk stratification, and management of systemic light-chain amyloidosis. Acta Haematol. 2019;141(2):93-106. https://doi.org/10.1159/000495455

17. Muchtar E, Gertz MA, Kumar SK, et al. Improved outcomes for newly diagnosed AL amyloidosis between 2000 and 2014: cracking the glass ceiling of early death. Blood. 2017;129(15):2111-2119. https://doi.org/10.1182/ blood-2016-11-751628

18. Quarta CC, Solomon SD. Stabilizing transthyretin to treat ATTR cardiomyopathy. N Engl J Med. 2018;379(11):1083-1084. https://doi.org/10.1056/nejme1810074

19. Maurer MS, Schwartz JH, Gundapaneni B, et al. Tafamidis treatment for patients with transthyretin amyloid cardiomyopathy. N Engl J Med. 2018;379(11):1007-1016. https://doi.org/10.1056/nejmoa1805689

20. Bahlis NJ, Lazarus HM. Multiple myeloma-associated AL amyloidosis: is a distinctive therapeutic approach warranted? Bone Marrow Transplant. 2006;38(1):7-15. https://doi.org/10.1038/sj.bmt.1705395 